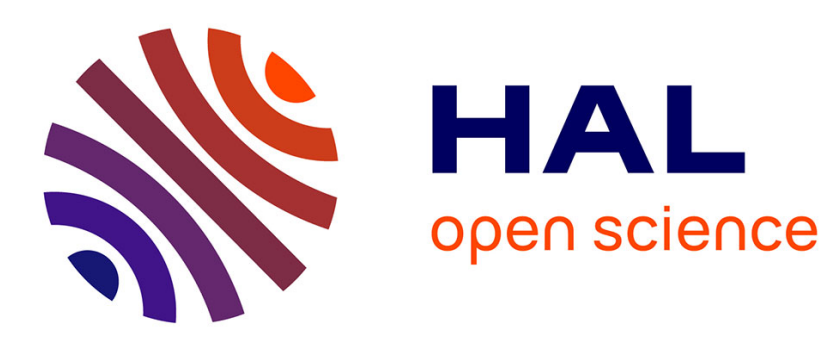

\title{
Edgeworth expansions for the product of two complex random matrices each with IID components
}

\author{
Christopher S. Withers, Saralees Nadarajah
}

\section{To cite this version:}

Christopher S. Withers, Saralees Nadarajah. Edgeworth expansions for the product of two complex random matrices each with IID components. Statistics and Probability Letters, 2010, 80 (23-24), pp.1954. 10.1016/j.spl.2010.08.025 . hal-00691778

\section{HAL Id: hal-00691778 \\ https://hal.science/hal-00691778}

Submitted on 27 Apr 2012

HAL is a multi-disciplinary open access archive for the deposit and dissemination of scientific research documents, whether they are published or not. The documents may come from teaching and research institutions in France or abroad, or from public or private research centers.
L'archive ouverte pluridisciplinaire HAL, est destinée au dépôt et à la diffusion de documents scientifiques de niveau recherche, publiés ou non, émanant des établissements d'enseignement et de recherche français ou étrangers, des laboratoires publics ou privés. 


\section{Accepted Manuscript}

Edgeworth expansions for the product of two complex random

matrices each with IID components

Christopher S. Withers, Saralees Nadarajah

PII:

S0167-7152(10)00248-8

DOI:

10.1016/j.spl.2010.08.025

Reference: $\quad$ STAPRO 5784

To appear in: Statistics and Probability Letters

Received date: 15 March 2010

Revised date: 24 August 2010

Accepted date: 25 August 2010

Please cite this article as: Withers, C.S., Nadarajah, S., Edgeworth expansions for the product of two complex random matrices each with IID components. Statistics and Probability Letters (2010), doi:10.1016/j.spl.2010.08.025

This is a PDF file of an unedited manuscript that has been accepted for publication. As a service to our customers we are providing this early version of the manuscript. The manuscript will undergo copyediting, typesetting, and review of the resulting proof before it is published in its final form. Please note that during the production process errors may be discovered which could affect the content, and all legal disclaimers that apply to the journal pertain. 


\title{
Edgeworth expansions for the product of two complex random matrices each with IID components
}

by

\author{
Christopher S. Withers \\ Applied Mathematics Group \\ Industrial Research Limited \\ Lower Hutt, NEW ZEALAND \\ Saralees Nadarajah \\ School of Mathematics \\ University of Manchester \\ Manchester M13 9PL, UK
}

\begin{abstract}
Let $\mathbf{A}$ and $\mathbf{B}$ be independent $n \times n$ complex matrices with elements i.i.d. as $X_{1}+i X_{2}$ and $Y_{1}+i Y_{2}$, respectively, where $i=(-1)^{1 / 2}, X_{1}$ and $X_{2}$ are independent and identically distributed, $Y_{1}$ and $Y_{2}$ are independent and identically distributed. We obtain Edgeworth expansions for the distribution of any element of $\mathbf{A B}$ (and so for trace $\mathbf{A B}$ ), in Cartesian coordinates. If $X_{1}$ or $Y_{1}$ is symmetric about zero, the expansions are given to $O\left(n^{-4}\right)$. A specific example applicable to reverberation systems is the case when $X_{1}$ and $Y_{1}$ are both Gaussian.
\end{abstract}

Keywords: Complex random matrices; Edgeworth expansions; Gaussian.

\section{Introduction and summary}

Let $\mathbf{A}, \mathbf{B}$ be independent $n \times n$ complex matrices with elements independent and identically distributed (i.i.d.) as

$$
A_{j k} \stackrel{\mathcal{L}}{=} X_{1}+i X_{2}, B_{j k} \stackrel{\mathcal{L}}{=} Y_{1}+i Y_{2},
$$

where $i=(-1)^{1 / 2}, X_{1}$ and $X_{2}$ are i.i.d. as $X, Y_{1}$ and $Y_{2}$ are i.i.d. as $Y$, and $X, Y$ are real random variables with finite moments. The need to know the distribution of the product, $\mathbf{A B}$, arises in many applied areas. An early problem is in the study of reverberation systems: in systems with $n$ feedback speakers $\mathbf{A}$ is the transmission function of the room with the speakers, and B is that of the feedback system. Schroeder (1964, page 1720) notes that $\mathbf{A}$ (that is $X$ ) is Gaussian distributed by a Central Limit Theorem argument. Such products also arise with respect to the capacity of mobile multiple-antenna communication links (Marzetta and Hochwald, 1999; Abhayapala et al., 2003; Godavarti et al., 2003), dynamics of nearly periodic disordered multi-span beams (Bouzit and Pierre, 2000), and wave component analysis of energy flow in complex structures (Wester and Mace, 2005a, 2005b). 
The aim of this note is to obtain the distribution of $\mathbf{A B}$ and its trace. The distribution of trace $\mathbf{A B}$ is just that of $(\mathbf{A B})_{11}$ with the parameter $n$ replaced by $n^{2}$.

The results are organized as follows. In Section 2, we obtain the moments and cumulants of $\mathbf{A B}$ when $n=1$. In Section 3, we obtain Edgeworth expansions for the distribution of an arbitrary element of $\mathbf{A B}$ in Cartesian form. We make specific references to the mentioned example on reverberation systems: see Example 3.1 for specific results, where both $\mathbf{A}$ and $\mathbf{B}$ are assumed to be Gaussian.

Elements $(\mathbf{A B})_{j k}$ and $(\mathbf{A B})_{l m}$ are independent if and only if $j \neq l$ and $k \neq m$. Expansions for their joint distribution could be obtained for the case when they are dependent by a straightforward extension of the method given here. The same is true for the joint distribution of any fixed number of elements of $\mathbf{A B}$.

\section{Moments and cumulants}

An arbitrary element of $\mathbf{A B}$ may be written as

$$
\sum_{j=1}^{n}\left(X_{1 j}+i X_{2 j}\right)\left(Y_{1 j}+i Y_{2 j}\right)=S_{n U}+i S_{n V}
$$

where

$$
S_{n U}=\sum_{j=1}^{n} U_{j}, S_{n V}=\sum_{j=1}^{n} V_{j}, U_{j}=X_{1 j} Y_{1 j}-X_{2 j} Y_{2 j}, V_{j}=X_{2 j} Y_{1 j}+X_{1 j} Y_{2 j},
$$

$\left\{X_{k j}\right\}$ are i.i.d. as $X,\left\{Y_{k j}\right\}$ are i.i.d. as $Y$ and $\left\{X_{k j}\right\}$ is independent of $\left\{Y_{k j}\right\}$. So, $\left\{\left(\begin{array}{c}U_{j} \\ V_{j}\end{array}\right)\right\}$ are i.i.d. as $\left(\begin{array}{c}U_{1} \\ V_{1}\end{array}\right)$. We assume that $m_{r X}=E X^{r}$ and $m_{r Y}=E Y^{r}$ are finite for $r=1,2,3, \ldots$ Then $m_{r s}=E U_{1}^{r} V_{1}^{s}$ is finite for $r, s=1,2,3, \ldots$ and may be expressed in terms of $\left\{m_{r X}, m_{s Y}\right\}$ :

$$
\begin{aligned}
& m_{10}=E U_{1}=0, m_{01}=E V_{1}=2 m_{1 X} m_{1 Y}, \\
& m_{20}=E U_{1}^{2}=2 m_{2 X} m_{2 Y}-2 m_{1 X}^{2} m_{1 Y}^{2}, \\
& m_{02}=E V_{1}^{2}=2 m_{2 X} m_{2 Y}+2 m_{1 X}^{2} m_{1 Y}^{2}, \\
& m_{11}=E U_{1} V_{1}=0 .
\end{aligned}
$$

Swapping $\left(X_{1}, Y_{1}\right)$ with $\left(X_{2}, Y_{2}\right)$ we see that

$$
\left(U_{1}, V_{1}\right) \stackrel{\mathcal{L}}{=}\left(-U_{1}, V_{1}\right)
$$

so that $m_{j k}=\kappa_{j k}=0$ for $j$ odd, where $\kappa_{j k}$ is the corresponding joint cumulant. Also, $E \exp \left(s U_{1}\right)=E \exp (s X Y) E \exp (-s X Y)$, so

$$
m_{r 0}=\sum_{j=0}^{r}\left(\begin{array}{l}
r \\
j
\end{array}\right)(-1)^{j} m_{j X} m_{j Y} m_{r-j, X} m_{r-j, Y}
$$

and $E \exp \left(s V_{1}\right)=\{E \exp (s X Y)\}^{2}$, so

$$
m_{0 r}=\sum_{j=0}^{r}\left(\begin{array}{l}
r \\
j
\end{array}\right) m_{j X} m_{j Y} m_{r-j, X} m_{r-j, Y}
$$


and the cumulants of $U_{1}$ and $V_{1}$ are given by

$$
\kappa_{r 0}=\kappa_{r}\left(U_{1}\right)=\left\{1+(-1)^{r}\right\} \kappa_{r}(X Y), \kappa_{0 r}=\kappa_{r}\left(V_{1}\right)=2 \kappa_{r}(X Y) .
$$

To obtain $\kappa_{r}(X Y)$ one uses the usual formula in terms of the moments, in this case $E(X Y)^{r}=m_{r X} m_{r Y}=t_{r}$ say. This can be written as

$$
\kappa_{r}(X Y)=\sum_{j=1}^{r}(-1)^{j-1}(j-1) ! B_{r j}(\mathbf{t}),
$$

where $B_{r j}(\mathbf{t})$ is tabled on page 307 of Comtet (1974) up to $r=12$, and is called the partial ordinary Bell polynomial for the sequence $\mathbf{t}=\left(t_{1}, t_{2}, \ldots\right)$ : see also Withers and Nadarajah (2009).

The Edgeworth expansions up to $O\left(n^{-1}\right)$ and $O\left(n^{-5}\right)$ require the cross-cumulants of $\left(U_{1}, V_{1}\right)$ up to order three and eight, respectively. These are given in Appendix A for the general case up to order three and for the case of $X$ or $Y$ symmetric about zero up to order eight.

However, simpler methods for obtaining these are available in special cases using

$$
E \exp \left(u U_{1}+v V_{1}\right)=E M_{Y}\left(u X_{1}+v X_{2}\right) M_{Y}\left(v X_{1}-u X_{2}\right),
$$

where $M_{Y}(s)=E \exp (s Y)$.

Example 2.1 Suppose $Y \sim \mathcal{N}(0,1)$. Then $M_{Y}(s)=\exp \left(s^{2} / 2\right)$, so the right hand side of (2.2) is equal to $E \exp (Q / 2)$ for $Q=2 T\left(X_{1}^{2}+X_{2}^{2}\right)$ and $2 T=u^{2}+v^{2}$. So,

$$
E \exp (Q / 2)=\sum_{r=0}^{\infty} M_{r} T^{r} / r !
$$

where

$$
M_{r}=E\left(X_{1}^{2}+X_{2}^{2}\right)^{r}=\sum_{j=0}^{r}\left(\begin{array}{l}
r \\
j
\end{array}\right) m_{2 j} m_{2 r-2 j} .
$$

Expanding gives

$$
m_{2 j, 2 k}=2^{-r} M_{r}(2 j) !(j !)^{-1}(2 k) !(k !)^{-1},
$$

where $r=j+k$, and other moments being zero. So,

$$
\left(U_{1}, V_{1}\right) \stackrel{\mathcal{L}}{=}\left(V_{1}, U_{1}\right)
$$

even if $X$ is not symmetric. Also the log of the right hand side of (2.2) is equal to

$$
2 \log E \exp \left(T X^{2}\right)=2 \sum_{r=1}^{\infty} \kappa_{r}\left(X^{2}\right) T^{r} / r !
$$

Expanding gives

$$
\kappa_{2 j, 2 k}=\kappa_{r}\left(X^{2}\right) 2^{1-r}(r !)^{-1}\left(\begin{array}{l}
r \\
k
\end{array}\right)(2 j) !(2 k) !,
$$

where $r=j+k$, and other cumulants being zero. Since $\left(U_{1}, V_{1}\right)$ is not altered by swapping $\left(X_{1}, X_{2}\right)$ with $\left(Y_{1}, Y_{2}\right)$, this also cover the case when $X \sim \mathcal{N}(0,1)$ and $Y$ is arbitrary. 
Example 2.2 Suppose both $X$ and $Y$ are $\mathcal{N}(0,1)$. Then $X_{1}^{2}+X_{2}^{2}$ is $\chi_{2}^{2}, E \exp \left(t \chi_{2}^{2}\right)=$ $(1-2 t)^{-1}$, so $M_{r}=2^{r} r$ ! and $\kappa_{r}\left(X^{2}\right) 2^{1-r}(r !)^{-1}=r^{-1}$, so (2.3) and (2.4) simplify to

$$
m_{2 j, 2 k}=r \kappa_{2 j, 2 k}=\left(\begin{array}{c}
r \\
j
\end{array}\right)(2 j) !(2 k) !
$$

where $r=j+k$. So, $m_{2 r, 0}=(2 r) !, \kappa_{2 r, 0}=2(2 r-1) !, \kappa_{20}=2, \kappa_{40}=12, \kappa_{60}=240$, $\kappa_{80}=10080, \kappa_{22}=4, \kappa_{42}=48, \kappa_{62}=1440, \kappa_{44}=864$, and so on.

Example 2.3 Similarly, one may show that if $X \sim \mathcal{N}\left(\mu_{X}, v_{X}\right)$ and $Y \sim \mathcal{N}\left(\mu_{Y}, v_{Y}\right)$ then the c.g.f. of $(U, V)$ is

$$
K_{U, V}(u, v)=-\log \left(1-2 T v_{X} v_{Y}\right)+\left(1-2 T v_{X} v_{Y}\right)^{-1}\left(\tau+2 v m_{X} m_{Y}\right)-\tau,
$$

where $2 T=u^{2}+v^{2}$ and $\tau=m_{X}^{2} / v_{X}+m_{Y}^{2} / v_{Y}$. So, $\kappa_{2 i, 2 j}=\lambda_{i j}\left(r^{-1}+\tau\right)$ for $r=i+j \geq 1$, and $\kappa_{2 i, 2 j+1}=(2 j+1) \lambda_{i j}\left(2 m_{X} m_{Y}\right)$ for $r=i+j \geq 1$, where $\lambda_{i j}=r !(2 i) !(2 j) !\left(v_{X} v_{Y}\right)^{r} /\{i !(r-i) !\}$ for $r=i+j$.

Example 2.4 Suppose that $X \sim$ Poisson $(\lambda)$. Then one may show that the joint noncentral moments of $(U, V)$ are

$$
m_{r s}=E \sum_{k=0}^{r+s} \lambda^{k} B_{r s, k}(\mathbf{A})
$$

where $\mathbf{A}=\left\{A_{r s}\right\}, A_{r s}=Y_{1}^{r} Y_{2}^{s}+Y_{1}^{s}\left(-Y_{2}\right)^{r}$, and $B_{r s, k}(\mathbf{A})$ is the partial exponential bivariate Bell polynomial. So, one obtains $\kappa_{r 0}=0$ for $r$ odd,

$$
\begin{aligned}
\kappa_{20}= & 2 \lambda m_{2 Y}+2 \lambda^{2} \kappa_{2 Y}, \\
\kappa_{40}= & 2 \lambda m_{4 Y}+2 \lambda^{2}\left(7 m_{4 Y}+m_{2 Y}^{2}-4 m_{1 Y} m_{3 Y}\right) \\
& \quad+4 \lambda^{3}\left(3 m_{4 Y}+m_{2 Y}^{2}-6 m_{3 Y} m_{1 Y}+2 m_{2 Y} m_{1 Y}^{2}\right) \\
& \quad+2 \lambda^{4}\left(m_{4 Y}+m_{2 Y}^{2}-4 m_{3 Y} m_{1 Y}+2 m_{2 Y} m_{1 Y}^{2}-2 m_{1 Y}^{4}\right), \\
\kappa_{01}= & 2 \lambda m_{1 Y}, \\
\kappa_{02}= & \kappa_{20}, \\
\kappa_{11}= & 0, \\
\kappa_{03}= & 2 \lambda m_{3 Y}+6 \lambda^{2}\left(m_{3 Y}-m_{2 Y} m_{1 Y}\right)+2 \lambda^{3} \mu_{3 Y}, \\
\kappa_{21}= & 2 \lambda m_{2 Y} m_{1 Y}+2 \lambda^{2}\left(m_{3 Y}-m_{2 Y} m_{1 Y}\right)+2 \lambda^{3} \mu_{3 Y},
\end{aligned}
$$

and so on.

\section{Cartesian Edgeworth expansions}

As noted in (2.1), an arbitrary element of $\mathbf{A B}$ can be written as

$$
S_{n U}+i S_{n V}=\sum_{j=1}^{n}\left(U_{j}+i V_{j}\right),
$$


where $\left(\begin{array}{c}U_{j} \\ V_{j}\end{array}\right)$ are i.i.d. with cumulants $\left\{\kappa_{r s}\right\}$ given by Section 2. In particular, $\kappa_{10}=0$, $\kappa_{01}=2 m_{1 X} m_{1 Y}, \kappa_{20}=\kappa_{02}=2\left(m_{2 X} m_{2 Y}-m_{1 X}^{2} m_{1 Y}^{2}\right)$, and $\kappa_{11}=0$. So, as $n \rightarrow \infty$,

$$
\mathbf{Y}_{n}=\left(n \kappa_{20}\right)^{-1 / 2}\left(\begin{array}{ll}
S_{n U} & \\
S_{n V} & -n \kappa_{01}
\end{array}\right) \stackrel{\mathcal{L}}{\rightarrow}\left(\begin{array}{l}
N_{1} \\
N_{2}
\end{array}\right)
$$

where $N_{1}$ and $N_{2}$ are i.i.d. $\mathcal{N}(0,1)$ random variables. Also by Bhattacharya and Rao (1976), if $X$ and $Y$ are nonlattice, then so is $\left(U_{1}, V_{1}\right)$, so $\mathbf{Y}_{n}$ has the Edgeworth expansions

$$
\begin{aligned}
& P_{\mathbf{Y}_{n}}(\mathbf{y})=P\left(\mathbf{Y}_{n} \leq \mathbf{y}\right)=\sum_{j=0}^{\infty} n^{-j / 2} P_{j}(\mathbf{y}) \\
& p_{\mathbf{Y}_{n}}(\mathbf{y})=\partial^{2} P_{\mathbf{Y}_{n}}(\mathbf{y}) / \partial y_{1} \partial y_{2}=\sum_{j=0}^{\infty} n^{-j / 2} p_{j}(\mathbf{y})
\end{aligned}
$$

where

$$
\begin{aligned}
& P_{j}(\mathbf{y})=\widetilde{P}_{j}(-\partial / \partial \mathbf{y}) \Phi\left(y_{1}\right) \Phi\left(y_{2}\right) \\
& p_{j}(\mathbf{y})=\partial^{2} P_{j}(\mathbf{y}) / \partial y_{1} \partial y_{2}=\widetilde{P}_{j}(-\partial / \partial \mathbf{y}) \phi\left(y_{1}\right) \phi\left(y_{2}\right),
\end{aligned}
$$

where $\Phi(\cdot)$ and $\phi(\cdot)$ are the distribution and density of a standard normal, and $\widetilde{P}_{j}(\mathbf{t})$ are certain polynomials in $\mathbf{t}=\left(t_{1}, t_{2}\right)$. In particular,

$$
\widetilde{P}_{0}(\mathbf{t})=1, \quad \widetilde{P}_{1}=\nabla_{1}, \widetilde{P}_{2}=\nabla_{2}+\nabla_{1}^{2} / 2, \widetilde{P}_{3}=\nabla_{3}+\nabla_{2} \nabla_{1}+\nabla_{1}^{3} / 6, \ldots,
$$

where

$$
\nabla_{j}(\mathbf{t})=\sum_{r+s=j+2} t_{1}^{r} t_{2}^{s} K_{r s}, K_{r s}=\kappa_{20}^{-(r+s) / 2} \kappa_{r s} /(r ! s !)
$$

So,

$$
\widetilde{P}_{1}(\mathbf{t})=\sum_{r+s=3} t_{1}^{r} t_{2}^{s} K_{r s}=t_{1}^{3} K_{30}+t_{1}^{2} t_{2} K_{21}+t_{1} t_{2}^{2} K_{12}+t_{2}^{3} K_{03}
$$

and

$$
p_{1}(\mathbf{y})=\left(H_{31} K_{30}+H_{21} H_{12} K_{21}+H_{12} H_{22} K_{12}+H_{32} K_{03}\right) \phi\left(Y_{1}\right) \phi\left(Y_{2}\right),
$$

where $H_{j k}=H_{j}\left(y_{k}\right)$ and $H_{j}(x)=\phi(x)^{-1}(-\partial / \partial x)^{j} \phi(x)$, the $j$ th Hermite polynomial, expressable as $H_{j}(x)=\operatorname{Re} E(x+i \mathcal{N}(0,1))^{j}$. Similarly,

$$
P_{1}(\mathbf{y})=-\left(H_{21} \phi_{1} \Phi_{2} K_{30}+H_{22} \Phi_{1} \phi_{2} K_{03}+H_{11} \phi_{1} \phi_{2} K_{21}+H_{12} \phi_{1} \phi_{2} K_{12}\right),
$$

where $\phi_{i}=\phi\left(y_{i}\right)$ and $\Phi_{i}=\Phi\left(y_{i}\right)$. Higher order $p_{j}(\mathbf{y})$ and $P_{j}(\mathbf{y})$ can be written down similarly.

Now suppose that $X$ is symmetric about zero. Then by Appendix A, $\kappa_{r s}=0$ unless both $r$ and $s$ are even, so $\widetilde{P}_{j}=\nabla_{j}=0$ for $j$ odd, $\widetilde{P}_{2}=\nabla_{2}, \widetilde{P}_{4}=\nabla_{4}+\nabla_{2}^{2} / 2, \widetilde{P}_{6}=$ $\nabla_{6}+\nabla_{4} \nabla_{2}+\nabla_{2}^{3} / 6$, and so on. Also $\kappa_{r s}=\kappa_{s r}$, so

$$
\begin{aligned}
& \widetilde{P}_{2}(\mathbf{t})=\nabla_{2}(\mathbf{t})=\sum^{2} t_{1}^{4} K_{40}+t_{1}^{2} t_{2}^{2} K_{22}, \\
& \nabla_{4}(\mathbf{t})=\sum^{2}\left(t_{1}^{6} K_{60}+t_{1}^{4} t_{2}^{2} K_{42}\right) \\
& \nabla_{6}(\mathbf{t})=\sum^{2}\left(t_{1}^{8} K_{80}+t_{1}^{6} t_{2}^{2} K_{62}\right)+t_{1}^{4} t_{2}^{4} K_{44},
\end{aligned}
$$


where

$$
\sum^{2} f\left(t_{1}, t_{2}\right)=f\left(t_{1}, t_{2}\right)+f\left(t_{2}, t_{1}\right)
$$

So,

$$
\widetilde{P}_{4}(\mathbf{t})=\sum^{2}\left(t_{1}^{8} P_{80}+t_{1}^{6} t_{2}^{2} P_{62}+t_{1}^{6} K_{60}+t_{1}^{4} t_{2}^{2} K_{42}\right)+t_{1}^{4} t_{2}^{4} P_{44}
$$

where

$$
P_{80}=K_{40}^{2} / 2, P_{62}=K_{40} K_{22}, P_{44}=K_{40}^{2}+K_{22}^{2} / 2,
$$

and

$$
\widetilde{P}_{6}(\mathbf{t})=\sum_{+t_{1}^{4} t_{2}^{4} K_{44}}^{2}\left(t_{1}^{12} P_{12,0}+t_{1}^{10} t_{2}^{2} P_{10,2}+t_{1}^{8} t_{2}^{4} P_{84}+t_{1}^{10} P_{10,0}+t_{1}^{8} t_{2}^{2} P_{82}+t_{1}^{6} t_{2}^{4} P_{64}+t_{1}^{8} K_{80}\right) t_{1}^{6} t_{2}^{6} P_{66}
$$

where

$$
\begin{aligned}
& P_{12,0}=K_{40}^{3} / 6, P_{10,2}=K_{40}^{2} K_{22} / 2, P_{84}=\left(K_{40}^{3}+K_{40} K_{22}^{2}\right) / 2, \\
& P_{10,0}=K_{60} K_{40}, P_{82}=K_{42} K_{40}+K_{60} K_{22} \\
& P_{64}=K_{60} K_{40}+K_{42} K_{40}+K_{42} K_{22} \\
& P_{66}=K_{22}^{3} / 6+6 K_{40}^{2} K_{22} .
\end{aligned}
$$

So,

$$
p_{\mathbf{Y}_{n}}(\mathbf{y})=\sum_{j=0}^{\infty} n^{-j} p_{2 j}(\mathbf{y})
$$

and

$$
P\left(\mathbf{Y}_{n} \leq \mathbf{y}\right)=\sum_{j=0}^{\infty} n^{-j} P_{2 j}(\mathbf{y})
$$

where $P_{0}(\mathbf{y})=\Phi_{1} \Phi_{2}, p_{0}(\mathbf{y})=\phi_{1} \phi_{2}$, and, for $r=2,4, \ldots$,

$$
P_{r}(\mathbf{y})=\left\{\sum^{2} a_{r}(\mathbf{y})+b_{r}(\mathbf{y})\right\} \phi_{1} \phi_{2}
$$

and

$$
P_{r}(\mathbf{y})=-\sum^{2} A_{r}(\mathbf{y}) \Phi_{1} \Phi_{2}+\left\{B_{r}(\mathbf{y})+\sum^{2} C_{r}(\mathbf{y})\right\} \phi_{1} \phi_{2}
$$

where

$$
\sum^{2} f(\mathbf{y})=f\left(y_{1}, y_{2}\right)+f\left(y_{2}, y_{1}\right)
$$


In particular,

$$
\begin{aligned}
a_{2}(\mathbf{y})= & H_{41} K_{40}, b_{2}(\mathbf{y})=H_{21} H_{22} K_{22}, \\
A_{2}(\mathbf{y})= & H_{31} K_{40}, B_{2}(\mathbf{y})=H_{11} H_{12} K_{22}, C_{2}(\mathbf{y})=0, \\
a_{4}(\mathbf{y})= & H_{81} P_{80}+H_{61} K_{60}+H_{61} H_{22} P_{62}+H_{41} H_{22} K_{42}, \\
b_{4}(\mathbf{y})= & H_{41} H_{42} P_{44}, \\
A_{4}(\mathbf{y})= & H_{71} P_{80}+H_{51} K_{60}, B_{4}(\mathbf{y})=H_{31} H_{32} P_{44}, \\
C_{4}(\mathbf{y})= & H_{51} H_{12} P_{62}+H_{31} H_{12} K_{42}, \\
a_{6}(\mathbf{y})= & H_{12,1} P_{12,0}+H_{10,1} H_{22} P_{10,2}+H_{81} H_{42} P_{84} \\
& +H_{10,1} P_{10,0}+H_{81} H_{22} P_{82}+H_{61} H_{42} P_{64}+H_{81} K_{80}, \\
b_{6}(\mathbf{y})= & H_{61} H_{62} P_{66}+H_{41} H_{42} K_{44}, \\
A_{6}(\mathbf{y})= & H_{11,1} P_{12,0}+H_{91} P_{10,0}+H_{71} K_{80}, \\
B_{6}(\mathbf{y})= & H_{51} H_{52} P_{66}+H_{31} H_{32} K_{44}, \\
C_{6}(\mathbf{y})= & H_{91} H_{12} P_{10,2}+H_{71} H_{32} P_{84}+H_{71} H_{12} P_{82}+H_{51} H_{32} P_{64},
\end{aligned}
$$

and so on.

Note 3.1 We have $p_{\mathbf{Y}_{n}}(\mathbf{y})=p_{\mathbf{Y}_{n}}\left(y_{1},-y_{2}\right)=p_{\mathbf{Y}_{n}}\left(-y_{1}, y_{2}\right)$, so $\mathbf{Z}_{n}=\left(\left|Y_{n 1}\right|,\left|Y_{n 2}\right|\right)^{\prime}$ has density

$$
p_{\mathbf{Z}_{n}}(\mathbf{y})=4 p_{\mathbf{Y}_{n}}(\mathbf{y})
$$

on $(0, \infty)^{2}$ and

$$
P\left(\mathbf{Z}_{n} \leq \mathbf{y}\right)=4\left\{P_{\mathbf{Y}_{n}}(\mathbf{y})-P_{\mathbf{Y}_{n}}\left(y_{1}, 0\right)-P_{\mathbf{Y}_{n}}\left(0, y_{2}\right)-P_{\mathbf{Y}_{n}}(\mathbf{0})\right\} .
$$

Also $P_{\mathbf{Y}_{n}}(\mathbf{0})=1 / 4$ and $p_{\mathbf{Z}_{n}}(\mathbf{y})=p_{\mathbf{Z}_{n}}\left(y_{2}, y_{1}\right)$.

Example 3.1 Suppose $X$ and $Y$ are $\mathcal{N}(0,1)$. Then by Example 2.2

$$
K_{2 j, 2 k}=2^{-j-k}(j+k)^{-1}\left(\begin{array}{c}
j+k \\
j
\end{array}\right) .
$$

In particular, $K_{40}=2^{-3}, K_{60}=2^{-3} / 3, K_{80}=2^{-6}, K_{22}=2^{-2}, K_{42}=2^{-3}, K_{62}=2^{-3}$, $K_{44}=3 \cdot 2^{-5}, P_{80}=2^{-7}, P_{62}=2^{-5}, P_{44}=3 \cdot 2^{-6}, P_{12,0}=2^{-10} / 3, P_{10,2}=2^{-9}$, $P_{84}=5 \cdot 2^{-10}, P_{10,0}=2^{-6} / 3, P_{82}=5 \cdot 2^{-6} / 3, P_{64}=5 \cdot 2^{-5} / 3$ and $P_{66}=5 \cdot 2^{-6} / 3$. So,

$$
\mathbf{Y}_{n}=(2 n)^{-1 / 2}\left(S_{n U}, S_{n V}\right)^{\prime}
$$


satisfies (3.1)-(3.4) with

$$
\begin{aligned}
a_{2}(\mathbf{y})= & 2^{-3} H_{41}, b_{2}(\mathbf{y})=2^{-2} H_{21} H_{22}, \\
A_{2}(\mathbf{y})= & 2^{-3} H_{31}, B_{2}(\mathbf{y})=2^{-2} H_{11} H_{12}, C_{2}(\mathbf{y})=0, \\
a_{4}(\mathbf{y})= & 2^{-7} H_{81}+2^{-5} H_{61}\left(4 / 3+H_{22}\right)+2^{-3} H_{41} H_{22}, \\
b_{4}(\mathbf{y})= & 3 \cdot 2^{-6} H_{41} H_{42}, \\
A_{4}(\mathbf{y})= & 2^{-7} H_{71}+2^{-3} H_{51} / 3, B_{4}(\mathbf{y})=3 \cdot 2^{-6} H_{31} H_{32}, \\
C_{4}(\mathbf{y})= & 2^{-5} H_{41} H_{12}+2^{-3} H_{31} H_{12}, \\
a_{6}(\mathbf{y})= & 2^{-10} H_{12,1} / 3+2^{-9} H_{10,1} H_{22}+5 \cdot 2^{-10} H_{81} H_{42} \\
& +10^{-6} H_{10,1} / 3+5 \cdot 2^{-6} H_{81} H_{22} / 3+5 \cdot 2^{-6} H_{61} H_{42} / 3+2^{-6} H_{81}, \\
b_{6}(\mathbf{y})= & 5 \cdot 2^{-3} H_{61} H_{62} / 3+3 \cdot 2^{-5} H_{41} H_{42}, \\
A_{6}(\mathbf{y})= & 2^{-10} H_{11,1} / 3+10^{-6} H_{91} / 3+2^{-6} H_{71}, \\
B_{6}(\mathbf{y})= & 5 \cdot 2^{-3} H_{51} H_{52} / 3+2^{-3} H_{31} H_{32}, \\
C_{6}(\mathbf{y})= & 2^{-9} H_{91} H_{12}+5 \cdot 2^{-10} H_{71} H_{32}+5 \cdot 2^{-6} H_{71} H_{12} / 3+5 \cdot 2^{-6} H_{51} H_{32} / 3 .
\end{aligned}
$$

\section{Appendix A}

Here, we give expressions for the joint moments $m_{r s}=E U^{r} V^{s}$ and corresponding cumulants $\kappa_{r s}$ for $U=X_{1} Y_{1}-X_{2} Y_{2}$ and $V=X_{2} Y_{1}+X_{1} Y_{2}$, where $X_{1}, X_{2} \stackrel{\mathcal{L}}{=} X$ and $Y_{1}, Y_{2} \stackrel{\mathcal{L}}{=} Y$ and $X_{1}, X_{2}, Y_{1}, Y_{2}$ are independent real random variables with finite moments $m_{r X}=E X^{r}$, $m_{r Y}=E Y^{r}$ for $r=1,2, \ldots$ In Section 2, we showed that $m_{r s}=\kappa_{r s}=0$ for $r$ odd, and gave expressions for general $m_{r 0}, m_{0 r}$. So,

$$
\begin{aligned}
& \kappa_{10}=m_{10}=0, \kappa_{01}=m_{01}=2 m_{1 X} m_{1 Y}, \\
& \kappa_{20}=m_{20}=\kappa_{02}=2\left(m_{2 X} m_{2 Y}-m_{1 X}^{2} m_{1 Y}^{2}\right), \\
& \kappa_{11}=m_{11}=0, \kappa_{30}=m_{30}=0, \kappa_{12}=m_{12}=0 .
\end{aligned}
$$

Also

$$
m_{21}=2\left(m_{1 X} m_{2 X} m_{3 Y}-2 m_{1 X} m_{2 X} m_{1 Y} m_{2 Y}+m_{3 X} m_{1 Y} m_{2 Y}\right),
$$

So

$$
\kappa_{21}=2\left(m_{1 X} m_{2 X} m_{3 Y}-4 m_{1 X} m_{2 X} m_{1 Y} m_{2 Y}+m_{3 X} m_{1 Y} m_{2 Y}+2 m_{1 X}^{3} m_{1 Y}^{3}\right) .
$$

This gives all the cumulants needed for the Edgeworth expansions up to $O\left(n^{-1}\right)$.

We now assume $Y$ is symmetric about zero, or equivalently, $X$ is symmetric about zero. We give those cumulants needed for the Edgeworth expansions up to $O\left(n^{-5}\right)$. So, $(U, V) \stackrel{\mathcal{L}}{=}\left(X_{1} Y_{1}+X_{2} Y_{2}, X_{2} Y_{1}-X_{1} Y_{2}\right) \stackrel{\mathcal{L}}{=}(V, U)$. So, $m_{r s}=m_{s r}, \kappa_{r s}=\kappa_{s r}, m_{r s}=\kappa_{r s}=0$ unless both $r$ and $s$ are even,

$$
m_{2 r, 0}=\sum_{j=0}^{r}\left(\begin{array}{l}
2 r \\
2 j
\end{array}\right) m_{2 j, X} m_{2 j, Y} m_{2 r-2 j, X} m_{2 r-2 j, Y}
$$


and

$$
\begin{aligned}
m_{2 r, 2 s}= & \sum_{j=0}^{2 r}\left(\begin{array}{c}
2 r \\
j
\end{array}\right) \sum_{k=0}^{2 s}\left(\begin{array}{c}
2 s \\
k
\end{array}\right) m_{j+2 s-k, X} m_{2 r-j+k, X} m_{j+k, Y} m_{2 r-j+2 s-k, Y} \\
& \times\{I(j, k \text { even })-I(j, k \text { odd })\}
\end{aligned}
$$

where $I(A)=1$ or 0 for A true or false. So,

$$
\begin{aligned}
& m_{20}=2 m_{2 X} m_{2 Y}, m_{40}=2\left(m_{4 X} m_{4 Y}+3 m_{2 X}^{2} m_{2 Y}^{2}\right), \\
& m_{60}=2\left(m_{6 X} m_{6 Y}+15 m_{4 X} m_{2 X} m_{4 Y} m_{2 Y}\right), \\
& m_{80}=2\left(m_{8 X} m_{8 Y}+28 m_{6 X} m_{2 X} m_{6 Y} m_{2 Y}+35 m_{4 X}^{2} m_{4 Y}^{2}\right), \\
& m_{22}=2\left(\sum^{2} m_{2 X}^{2} m_{4 Y}-2 m_{2 X}^{2} m_{2 Y}^{2}\right), \\
& m_{42}=2\left(\sum^{2} m_{4 X} m_{2 X} m_{6 Y}-2 m_{4 X} m_{2 X} m_{4 Y} m_{2 Y}\right), \\
& m_{62}=2\left(\sum^{2} m_{8 X} m_{6 Y} m_{2 Y}-12 m_{6 X} m_{2 X} m_{6 Y} m_{2 Y}+15 \sum m_{6 X} m_{2 X} m_{4 Y}-20 m_{4 X}^{2} m_{4 Y}^{2}\right), \\
& m_{44}=2\left\{\sum^{2} m_{4 X}^{2}\left(m_{8 Y}-16 m_{6 Y} m_{2 Y}\right)+12 m_{6 X} m_{2 X} m_{6 Y} m_{2 Y}+18 m_{4 X}^{2} m_{4 Y}^{2} m_{4 Y}^{2}\right\},
\end{aligned}
$$

where $\sum^{2} f(X, Y)=f(X, Y)+f(Y, X)$.

The condition that $Y \stackrel{\mathcal{L}}{=}-Y$ can be weakened. For example, (A.1) and (A.4) hold if $E Y=0,(\mathrm{~A} .2),(\mathrm{A} .3)$ and (A.5) hold and $\kappa_{21}=0$ if $E Y=E Y^{3}=0$, and so on. So, if $E Y=E Y^{3}=0$ (or $E X=E X^{3}=0$ ) then the error in the Central Limit Theorem approximation is $O\left(n^{-1}\right)$ not just $O\left(n^{-1 / 2}\right)$. 
So,

$$
\begin{aligned}
& \kappa_{20}=2 m_{2 X} m_{2 Y}, \\
& \kappa_{40}=2\left(m_{4 X} m_{4 Y}-3 m_{2 X}^{2} m_{2 Y}^{2}\right), \\
& \kappa_{60}=2\left(m_{6 X} m_{6 Y}-15 m_{4 X} m_{2 X} m_{4 Y} m_{2 Y}+30 m_{2 X}^{3} m_{2 Y}^{3}\right), \\
& \kappa_{80}=2 m_{8 X} m_{8 Y} \\
& +14\left(-4 m_{6 X} m_{2 X} m_{6 Y} m_{2 Y}-5 m_{4 X}^{2} m_{4 Y}^{2},+60 m_{4 X} m_{2 X} m_{4 Y} m_{2 Y}-90 m_{2 X}^{4} m_{2 Y}^{4}\right) \text {, } \\
& \kappa_{22}=\mu_{22}-\mu_{20} \mu_{02}-2 \mu_{11}^{2}=2\left(\sum^{2} m_{2 X}^{2} m_{4 Y}-4 m_{2 X}^{2} m_{2 Y}^{2}\right) \text {, } \\
& \kappa_{42}=\mu_{42}-6 \mu_{20} \mu_{22}-8 \mu_{11} \mu_{31}-\mu_{02} \mu_{40}+6 \mu_{20}^{2} \mu_{02}+24 \mu_{11}^{2} \mu_{20} \\
& =2\left\{\sum^{2} m_{4 X} m_{2 X}\left(m_{6 Y}-12 m_{2 Y}^{3}\right)-4 m_{4 X} m_{2 X} m_{4 Y} m_{2 Y}+42 m_{2 X}^{3} m_{2 Y}^{3}\right\}, \\
& \kappa_{62}=\mu_{62}-\mu_{02} \mu_{60}-12 \mu_{11} \mu_{51}-15 \mu_{20} \mu_{42}-15 \mu_{40} \mu_{22} \\
& -20 \mu_{31}^{2}+90 \mu_{22} \mu_{20}^{2}+30 \mu_{40}\left(\mu_{20} \mu_{02}+2 \mu_{11}^{2}\right) \\
& +240 \mu_{31} \mu_{11} \mu_{20}-270 \mu_{02} \mu_{20}^{3}-360 \mu_{11}^{2} \mu_{20}^{2} \\
& =2\left\{\sum^{2} m_{8 X} m_{6 Y} m_{2 Y}-14 m_{6 X} m_{2 X} m_{6 Y} m_{2 Y}+15 \sum^{2} m_{6 X} m_{2 X}\left(m_{4 Y}^{2}-2 m_{4 Y} m_{2 Y}^{2}\right)\right. \\
& -20 m_{4 X}^{2} m_{4 Y}^{2}+210 m_{4 X} m_{2 X}^{2} m_{4 Y} m_{2 Y}^{2}-30 \sum^{2} m_{4 X}^{2} m_{4 Y} m_{2 Y}^{2} \\
& \left.+270 \sum^{2} m_{4 X} m_{2 X}^{2} m_{2 Y}^{4}-2340 m_{2 X}^{4} m_{2 Y}^{4}\right\} \text {, } \\
& \kappa_{44}=\mu_{44}-6 \mu_{20} \mu_{24}-6 \mu_{02} \mu_{42}-16 \mu_{11} \mu_{33}-\mu_{40} \mu_{04}-16 \mu_{31} \mu_{13} \\
& -18 \mu_{22}^{2}+6 \mu_{40} \mu_{02}^{2}+6 \mu_{04} \mu_{20}^{2}+96 \mu_{11}\left(\mu_{31} \mu_{02}+\mu_{13} \mu_{20}\right) \\
& +72 \mu_{22}\left(\mu_{20} \mu_{02}+2 \mu_{11}^{2}\right)-18\left(\mu_{20}^{2} \mu_{02}^{2}+12 \mu_{20} \mu_{02} \mu_{11}^{2}+8 \mu_{11}^{4}\right) \\
& =2\left\{\sum^{2} m_{4 X}^{2}\left(m_{8 Y}-16 m_{6 Y} m_{2 Y}\right)+12 m_{6 X} m_{2 X} m_{6 Y} m_{2 Y}\right. \\
& +16 m_{4 X}^{2} m_{4 Y}^{2}-24 \sum^{2} m_{6 X} m_{2 X} m_{4 Y} m_{2 Y}^{2}+12 m_{4 X} m_{2 X}^{2} m_{4 Y} m_{2 Y}^{2} \\
& \left.-36 \sum^{2} m_{4 X}^{2} m_{2 Y}^{4}+432 \sum^{2} m_{4 X} m_{2 X}^{2} m_{2 Y}^{4}-738 m_{2 X}^{4} m_{2 Y}^{4}\right\} \text {. }
\end{aligned}
$$

\section{Acknowledgments}

The authors would like to thank the referee and the Editor for careful reading and for their useful comments.

\section{References}

[1] Abhayapala, T. D., Pollock, T. S. and Kennedy, R. A. (2003). Spatial decomposition of MIMO wireless channels. In: Proceedings of the 7th International Symposium on Signal Processing and Its Applications, volume I, pp. 309-312. 
[2] Bhattacharya, R. N. and Rao, R. R. (1976). Normal Approximation and Asymptotic Expansions. John Wiley and Sons, New York.

[3] Bouzit, D. and Pierre, C. (2000). Wave localization and conversion phenomena in multi-coupled multi-span beams. Chaos Solitons and Fractals, 11, 1575-1596.

[4] Comtet, L. (1974). Advanced Combinatorics. Reidel, Dordrecht.

[5] Godavarti, M., Marzetta, T. L. and Shamai, S. (2003). Capacity of a mobile multipleantenna wireless link with isotropically random Rician fading. IEEE Transactions on Information Theory, 49, 3330-3334.

[6] Gradshteyn, I. S. and Ryzhik, I. M. (2007). Table of Integrals, Series and Products, seventh edition. Academic Press, New York.

[7] Marzetta, T. L. and Hochwald, B. M. (1999). Capacity of a mobile multiple-antenna communication link in Rayleigh flat fading. IEEE Transactions on Information Theory, 45, 139-157.

[8] Schroeder, M. R. (1964). Improvement of acoustic-feedback stability by frequency shifting. Journal of the Acoustical Society of America, 36, 1718-1724.

[9] Wester, E. C. N. and Mace, B. R. (2005a). Wave component analysis of energy flow in complex structures - Part II: ensemble statistics. Journal of Sound and Vibration, 285, 229-250.

[10] Wester, E. C. N. and Mace, B. R. (2005b). Wave component analysis of energy flow in complex structures - Part III: two coupled plates. Journal of Sound and Vibration, 285, 251-265.

[11] Withers, C. S. and Nadarajah, S. (2009). Moments from cumulants and vice versa. International Journal of Mathematical Education in Science and Technology, 40, 842845. 